general health and with the pregnancy unharmed: About three weeks later the pain returned and ler general health again became impaired, and she was readmitted. There was, however, no recurrence of the pyrexia, and the patient picked up rapidly with rest in bed and proper dieting. She remained in hospital until full term, when a healthy child was born normally. Four days after delivery the urine was free from pus, and remained so up till now (three weeks later).

There was no difficulty about the diagnosis in this case. The acute onset of the pain, localized at first behind and below the right costal margin, and then spreading rapidly along the course of the right ureter, accompanied with pyrexia and pyuria, left no doubt as to the true nature of the disease. One very prominent feature of these cases is the somewhat diffuse area of great superficial tenderness, which seems to be confined to the cutaneous distribution of the anterior branch of the twelfth dorsal nerve. Pyelitis of pregnancy has to be distinguished in its early stages from pleurisy at the base of the right lung and in the later stages from appendicitis and enteric fever. The knowledge that pyelitis is a fairly common complication of pregnancy a careful inquiry into the history of the pain, and the fact that the tenderness is most marked behind and below the twelfth rib and along the course of the ureter, together with the intense hyperaesthesia, without much rigidity over the right side of the abdomen, will easily settle any doubt in most cases. The presence of pus in the urine will also give a definite clue to the condition, but it is not by any means safe to negative pyelitis if pus is absent from any given specimen. Several specimens must be examined, and it often happens that the pus does not appear in the urine until some days after the onset of the pain and pyrexia.

No doubt constipation plays a prominent part in the etiology of pyelitis, and almost without exception the infection is due to an invasion of the renal pelvis by the Bacillus coli. The renal pelvis is probably infected direct from the colon by means of the lymphatics. It is impossible to accept the ascending theory, because one cannot see why the infection should nearly always be right-sided, and, moreover, the bladder seldom shows signs of infection. In the few cases that I have seen in which the disease was ushered in with cystitis, the infection has been bilateral; and no doubt in these cases, which are far more serious, the infection was ascending. This condition is very different to what may be called simple pyelitis of pregnancy, which, as a rule, runs a very definite and satisfactory course.

The above case shows clearly what I have noted in many of these cases-namely, that the pyuria persists up till the time of delivery, but all the virulency appears to pass out of the organisms as soon as the acute stage is over. Nevertheless, the patients require a considerable amount of care after the acute stage is passed, and they still maintain the pale toxic look which is so characteristic of chronic coli absorption. The patient during this quiescent interval should lead an invalid's life. She should be kept warm, and take only verv gentle exercise, and ber food should consist mostly of milk and farinaceous stuffs. She should take no red meat, and very little fish and other white foods. Alcohol should be strictly avoided. Iron tonics and special attention to the bowels should be insisted upon, and occasional doses of urotropin given. She should accustom herself to drink a considerable quan. tity of water, especially in the early morning, for flushing purposes. One of the diuretic saline waters will probably be found usefal in combination with the urotropin.

She need not be confined to bed, but should take plenty of rest, preferably in the sunshine. If, after the acute symptoms have abated for some time, the patients are treated in the manner outlined above, the pregnancy will in all probability pass on to a normal delivery at term, and at the same time there need be little fear that there will be any permanent damage to the kidney. Should the pyuria persist after delivery, some permanent damage to the kidney substance must be suspected, and the pa.tient should be warned that she may have a recurrence of the disease in future pregnancies, which will possibly result in the formation of a pyonephrosis. One must also not neglect to search for a renal calculus or tubercle bacilli if the urine does not remain free from pus some time after the child is delivered. I have once seen a pyonephrosis develop during the second pregnancy in a patient who had had an attack of pyelitis at the fifth month of her first pregnancy, but recurrence in future pregnancies is a very rare exception.

With regard to the vaccine, it is, of course, impossible to. be sure whether the sudden drop of the temperature and the cessation of the pain in the above case was due to the vaccine or whether it was simply coincidence. I have seen improvement follow the injection of $10 \mathrm{cccm}$. coli vaccine in other cases of pyelitis, and have no doubt that this. treatment should be given a fair trial. 'If, however, the temperature and pain persist for more than ten days and infection appears to increase in virulency, in spite of the vaccine, dieting, and the administration of potassium iodide and urotropin, the uterus should be emptied.

On several occasions I have seen rapid recovery from pyelitis after spontaneous delivery. No doubt the presence of gestation in the uterus causes pressure on. the ureter and prevents free drainage.

The uncomplicated cases of pyelitis run such an even and satisfactory course and the signs and symptoms are so typical that any mistake in the diagnosis must be a matter for. regret. The greatest danger lies in mistaking this condition for appendicitis, and since the general appearance of the patient and the acute onset of pain and pyrexia are very similar in both diseases, a hasty conclusion may lead to a disastrous laparotomy.

If the symptoms are prolonged and the infection does not abate within a fortnight on suitable treatment, it. becomes a question as to whether the kidney substance itself has not become infected. In one case in which the kidney was explored we actually saw small abscesses in the kidney substance; and although the section of the kidney did no good, for there were many unopened abscess. cavities and drainage was impossible, the patient eventu. ally made an excellent recovery, and the pus completely disappeared from the urine after the birth of a living full. term child. If the kidney becomes infected, the possibility of renal pyaemia may be thought of ; but since the infection seldom affects both kidneys, there need be little fear of uraemia or anuria.

For practical clinical purposes I should divide the cases of simple coli pyelitis of pregnancy into three groups: (1) Mild, (2) moderate, and (3) severe. For the mild forms rest in bed, a milk diet, careful regulation of the bowels, and the administration of potassium iodide and urotropin will probably be sufficient. For the moderate the same treatment should be adopted, with the addition of a coli vaccine; and for the severe forms, when this treatment does not meet with success, the uterus should be emptied. It is not advisable to attempt to drain the pelvis of the kidney through the loin, because the patients cannot well stand the shock of the operation, and moneover, if the kidney itself is infected there will be many small foci which cannot be effectively dealt with. Bg far the safest way to effect free drainage is to relieve the pressure of the uterus upon the ureter. The labour, of course, should be set going by passing a sound or a bougie

\section{ACUTE INVERSION OF THE UTERUS.}

\section{By ALAN W. HOLTHUSEN, L.R.C.P., M.R.C.S.,}

HOUSE-SURGEON TO THE LEYTON, WALTHAMSTOW, AND WANGTEAD CHILDREN'S AND GENERAL HOSPITAL.

THE following case is of interest, not only on account of its rarity, but of other points to which attention will be drawn:

On August 23rd, 1908, I was called to Mrs. W., aged 31, a. primipara, who had been in labour for nine hours when seen at 5 a.m. The child was found to be in the fourth position of the vertex, with the head imperfectly flexed. At 8.30 a.m. the os was fully dilated, and an attempt was made to improve the flexion of the head. The head being still unrotated two hours later, Dr. S. J. J. Weakley was called in ; the forceps was. applied under chloroform; the head rotated easily, and the child was safely delivered in twenty-five minutes. The patient Jon recovered consciousness. The pulse-rate was then 108

Only one contraction of the uterus was noticed during the first half-hour after the birth of the child, and $1 \mathrm{c.cm}$. of ergotin was therefore given hypodermically. The patient was preventedi from bearing down during this period. The uterus remained soft for half an hour longer, when a sudden violent and painful contraction occurred. This was immediately followed by the: protrusion from the vulva of the inverted uterus, with the placenta attached. Very little haemorrhage had taken place placenta attached. The placenta was easily and quickly peeled up to this point. The placenta was easily and quickly peeled
off the inverted fundus, which was at once pushed back by the 
doubled-up fist. I sent for assistance, as the patient became suddenly very collapsed-pale, clammy, unconscious, the pulse at the wrist flickering and uncountable. The uterus was quite atonic, and immediately the hand was withdrawn from its cavity it reinverted. The haemorrhage was now considerable. In the absence of Dr. Weakley, Dr. E. B. Randall attended, and the patient was given a further $1 \mathrm{c} . \mathrm{cm}$. of - ergotin and and the patient was given a further 1 c.cm.. of ergotin and were infused into the right median basilic vein. Meanwhile the uterus was controlled as far as possible by bimanual compression. The patient gradually recovered from the shock; the uterus began to retract; so that at the end of half an hour the compression was no longer necessary. The patient recovered consciousness and inquired eagerly after the baby. Her pulserate at this point was 120 . The haemorrhage had now practically ceased.

The placenta was not morbidly adherent at any spot; no difficulty was experienced in detaching it from the fundus; it was of the "battledore" variety, and appeared to be centrally situated on the fundus. The cord was only $13 \frac{1}{2}$ in. long, and was coiled once round the child's body.

\section{AFTER-HISTORY}

The foot of the bed was raised on a chair. For the first four hours loz. of brandy was given every hour in an equal quantity of milk. The patient was also encouraged to drink as much water as she could. Later the stimulant was reduced, so that by the third day the patient was taking $2 \mathrm{oz}$. in twentyfour hours. A mixture was given containing ergot $m x v$ and hyoscyamus. The bowels were opened on the fourth day by is oz. of castor oil ; ordinary light diet was allowed after that day. The ergot was stopped on the sixth day; later a mixture was given containing ferri et amm. cit. gr. $x$ and tinct. nuc. vom. $m$ v.

The patient's recovery was uneventful; she got up nn the twenty-eighth day. The temperature did not rise above $98.8^{\circ} \mathrm{F}$. during the puerperium; the pulse-rate after the second day varied between 76 and 84 . The uterus involuted naturally; the lochia were scanty, and ceased on the eighth day; they were not otherwise abnormal in any way.

I saw the patient on October 20th, 1908; she was then in her usual good health.

I would call attention to this case for several reasons :

1. Its rarity. Acute inversion of the uterus occurred once in 190,000 labours at the Rotunda Hospital Dublin, ${ }^{1}$ and not once in 250,000 labours reported by Braun and Spaeth $;^{2} 1$ in 180,000 to 200,000 are the figures given by Eden. ${ }^{3}$

2. The extreme uterine inertia, whereby the uterine walls were so lax that, after the uterus had been replaced, it reinverted if the hand were withdrawn from its cavity.

3. The shortness of the cord, which was only $13 \frac{1}{2}$ in long; it was further artificially shortened by being coiled once round the child's body, giving a "practical" length of about 6 in.

4. The characteristic and excessive shock which occurred immediately after the inversion, before any haemorrhage occurred. The shock was out of all pro. portion to the amount of blood lost, which was probably less than 2 pints.

5. I would call special attention to the value of bimanual compression as a successful means of controlling post. partum haemorrhage while the uterus is regaining its tone.

I would suggest the following explanation of the events that took place. The explanation is based upon the theoretical considerations detailed in Champneys's epitome ${ }^{4}$ of Matthews Duncan's chapter on acute inversion. ${ }^{5}$ When the child was delivered by forceps, the fundus, owing to the extreme shortness of the cord, was unknowingly pulled upon. After the birth of the child the placenta and its site were further introverted into the cavity of the relaxed uterus by the weight of the child's body exerting traction on the cord. When the uterus did contract, the introverted placental site acted physiologically as a foreign body; it was, therefore, expelled by the contraction. The uterus then relaxed again; the placenta was removed, and consequently an ordinary post.partum haemorrhage occurred from the relaxed placental site, both before and after its reposition inside the body. This was eventually controlled by bimanual compression. The case would thus fall into the class of "artificial active inversion." 6

In conclusion, I have to acknowledge my indebtedness to Dr. S. J. J. Weakley and Dr. E. B. Randall, of Forest Gate, for the prompt and successful assistance they gave me.

1 Lusk Midwifery, 1896 REFERENCES.

1 Lusk, Midwifery, 1896 edition. 2 Loc. cit. ${ }^{3}$ Eden, Midwifery, 1906 edition. 4 St. Bartholomew's Hospital Journal, May, 1896. Merechanism of Natural and Morbid Labour, chap. xx. 6 Champneys, loc. cit.

\section{AN EXPERIMENT IN THE TREATMENT OF} EPILEPSY.

BY DAVID GOYDER, M.D.,

CONSULTING MEDICAL OFFICER TO THE BRADFORD ROTAL INFIRMARY CHAIRMAN OF THE HOSPITALS COMMITTEE, BRAUFORD POOR-LAW ENION.

The Bradford guardians decided to remove the sane epileptics from the workhouse to a house, Daisy Hill, in order to test the effect upon them of open-air residence with appropriate occupation, but with entire exclusion of medical treatment or the use of any drugs, it having been asserted that such residence alone would reduce the number of fits, and would probably lead to cure. While still at the workhouse, and on the initiative of Mr. Edward Milnes, the sane epileptics were separated from the rest, and under an attendant set to work in the gardens of the house, with the objects of improving their condition and rendering them useful. When I joined the gaardians, at the election of 1904, the method of treating epileptics by sodium bromide as salt with their food, and the exclusion of all common salt (chloride of sodium), had so impressed me, that I proposed the addition of this treatment in lieu of any other medicines for the sane epileptics whom $\mathbf{M r}$. Milnes had set to work on the grounds. The proposal was accepted and carried into effect by me. This treatment was continued until their removal to Daisy Hill, and with decidedly satisfactory results.

Upon their removal, and in view of the test as to the value of place, all medical treatment was abandoned, the hope being expressed that they would improve without medicine. During this period the personnel of the guardians was radically changed by the election of March, 1907, and Mr. Milnes's excellent supervision as a guardian was gone. Some time afterwards he begged me to keep an eye on the sane epileptics at Daisy Hil], and over a year ago I made systematic visits to Daisy Hill and took shorthand notes, of their condition. This supervision I have continued to the present time.

I found that the number of fits suffered by the men had not only largely increased, but month by month got progressively worse; as Chairman of the hospital committee I felt my responsibility for the condition of these men, and I took upon myself the duty of altering that condition. I made up my mind that this state of things and this test of place should at once be put an end to; and through Dr. Spark and Mr. Carter it was determined to see that the bromide salt treatment was strictly carrird into $\epsilon$ ffect. Dr. Spark is the accredited attendant at Daisy Hill, and under his supervision the new treatment, when determined upon, was cordially carried out.

The following table and comments show the conditions of things from January, 1907, to October of the same jear, at which date $I$ interven $\epsilon$ :

TABLE $I$.

\begin{tabular}{|c|c|c|c|c|}
\hline \multirow{2}{*}{$\begin{array}{l}\text { No. of } \\
\text { Mein. }\end{array}$} & \multicolumn{2}{|c|}{ Date. } & \multirow{2}{*}{ Fits. } & \multirow{2}{*}{ Increase. } \\
\hline & From & To & & \\
\hline 10 & Jan. 1, 1907 & Jan. 31, 1907 & 65 & \\
\hline 10 & Feb. 1, , & Mar. 12, , & 69 & (About 6 weeks.) \\
\hline 10 & Mar. 13, . & Apr. 13, ," & 58 & \\
\hline 10 & Apr. 14, ," & $\operatorname{May} 11,$, & 55 & \\
\hline 10 & May 12, , & June 8, ", & 80 & 15 \\
\hline 10 & June 9, , & July 6, " & 70 & . \\
\hline 9 & July 7, , & Aug. $3, \quad$, & 90 & 2 \\
\hline 9 & Aus. 4, ," & Aug. 31, :, & 90 & \\
\hline 9 & Sent. 1, ", & Sept. 28, ,, & 100 & 3 \\
\hline 9 & Sept. 29 " & Oct. 26, , , & 113 & 48 \\
\hline \multirow[t]{2}{*}{9} & Oct. 27, , & Nov. 23, & 108 & \\
\hline & & & 898 & in 11 months. \\
\hline
\end{tabular}

It will be observed that although from July to November the number of men was reduced to 9 , the increase in the 